\title{
Wideband Radar Cross Section Diagnostic Measurements
}

\author{
DEAN L. MENSA, MEMBER, IEEE
}

\begin{abstract}
This paper describes a diagnostic radar cross section (RCS) measurement system which uses a low-power, wideband, linear-FM radar to provide RCS responses of targets as a function of frequency, range, cross range, and angle. Range and frequency responses are produced by using an FFT analyzer and a desktop computer to perform on-line signal processing and provide rapid access to final results. Twodimensional maps of the target RCS distribution in range and cross range are obtained by off-line processing of recorded data. The system processes signals resulting from a swept bandwidth exceeding $3 \mathrm{GHz}$ to provide range resolution of less than $10 \mathrm{~cm}$. The various operating modes of the instrumentation provide a powerful tool for RCS diagnostic efforts in which individual scattering sources must be isolated and characterized. Several examples of results obtained from measurements of a number of simple and complex shapes are presented to demonstrate the utility and performance limits of the instrumentation.
\end{abstract}

\section{INTRODUCTION}

$\mathrm{E}$ CHOES backscattered from complex targets irradiated by short-wavelength radars can be characterized as superpositions of signals emanating from localized scattering centers on the target. By processing radar echoes, it is possible to determine the location and magnitude of individual scattering centers. When this is accomplished with adequate resolution, a radar image is obtained which provides a map of the spatial distribution of the target's reflectivity. Targets can be imaged by methods which independently yield resolution in range (along the propagation direction) and in cross range (normal to the propagation direction). Resolution in range is obtained by time-delay sorting of wideband signals, and resolution in cross range can be obtained by synthetic aperture methods [1], [2] .

The radar cross section (RCS) of a target, a measure of its scattering properties, is most often determined by measurements using calibrated microwave instrumentation systems. Conventional RCS measurements are performed by recording signals backscattered from targets irradiated by a continuouswave source. This provides a measure of the magnitude and phase of the total backscattered signal and, for a discrete aspect angle, the measured data are represented by a single complex number. A significant distinction for RCS measurements can be made in terms of resolution. In low-resolution measurements, the target is totally contained within the spatial resolution cell and no information can be obtained about the individual scattering centers. In high-resolution measurements, made by scanning a large target with a small resolution cell, a selective mapping of individual scattering centers can be obtained. Data obtained from this type of measurement provide

Manuscript received March 22, 1984.

The author is with the Pacific Missile Test Center, Point Mugu, CA. information on the spatial distribution of target reflectivity which aids the discovery of physical causes of the results observed in low-resolution measurements.

Fig. 1 shows examples of low- and high-resolution RCS data. The plot on the left is a conventional RCS pattern showing magnitude as a function of aspect angle for a particular frequency. The existence of multiple scatterers can be inferred from the rapid fluctuations, but individual scattering centers cannot be identified. The plot on the right shows the RCS as a function of range for a particular aspect angle obtained by a system which provides a high degree of resolution in range. The magnitude and location of individual scattering centers are clearly identified.

High-resolution backscatter information can be obtained by measuring the RCS of the target as a function of frequency for a fixed aspect angle. Measurements of this type can be made by either stepping or sweeping the frequency of the irradiating source. If the individual scattering centers on the target are relatively insensitive to frequency, the results of swept- or stepped-frequency measurements can be processed. to obtain the range distribution of the scattering centers. These data provide fundamental information about the spatial distribution of the backscattered sources. The range distribution of scattering centers can also be obtained by using a shortpulse radar, in which case the range resolution is determined by the pulse duration. The coherent data, obtained by varying the frequency over a bandwidth, can be recorded as a function of aspect angle and subsequently processed to resolve the target space in range and cross range. This provides a two-dimensional map of the spatial distribution of the scattering centers. By using wideband coherent signals and sophisticated signal processing, it is possible to determine the RCS of a target as a function of frequency, range, cross range, and angle by a single measurement set up. The ability to determine the location, magnitude, and character of scattering centers provides a diagnostic test function which is essential for efforts to alter the scattering properties of complex targets.

\section{System Description}

Three systems with center frequencies of $7.5,10$, and 15 $\mathrm{GHz}$ have been developed for the purpose of collecting highresolution RCS data in the controlled environments of the two anechoic chambers at the Pacific Missile Test Center. The microwave systems use linear-FM over a $3-\mathrm{GHz}$ bandwidth to obtain range resolution as high as $5 \mathrm{~cm}$. Resolution in cross range is obtained by processing coherent echoes measured as a function of target rotation. Signal-processing algorithms 
LOW RESOLUTION DATA

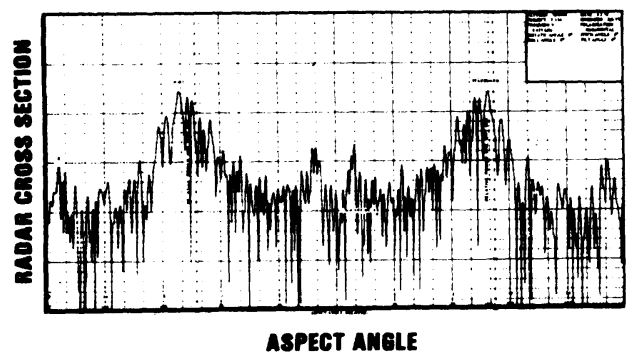

(a)

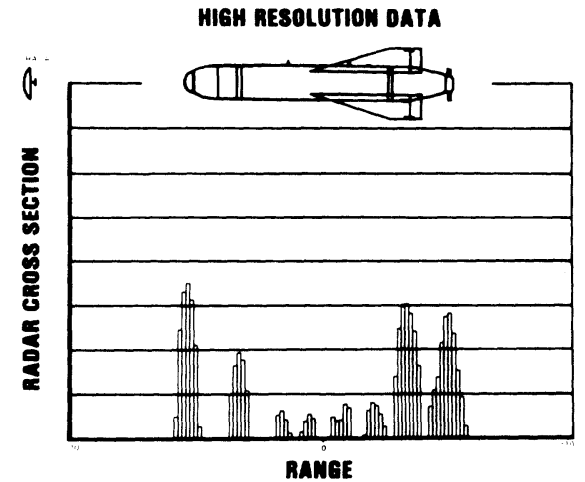

(b)

Fig. 1. Examples of low- and high-resolution RCS data. (a) The observed radar cross section is the phased superposition of signals from the scattering centers. Individual contributors cannot be identified.

(b) Contributions of individual scattering centers are isolated.

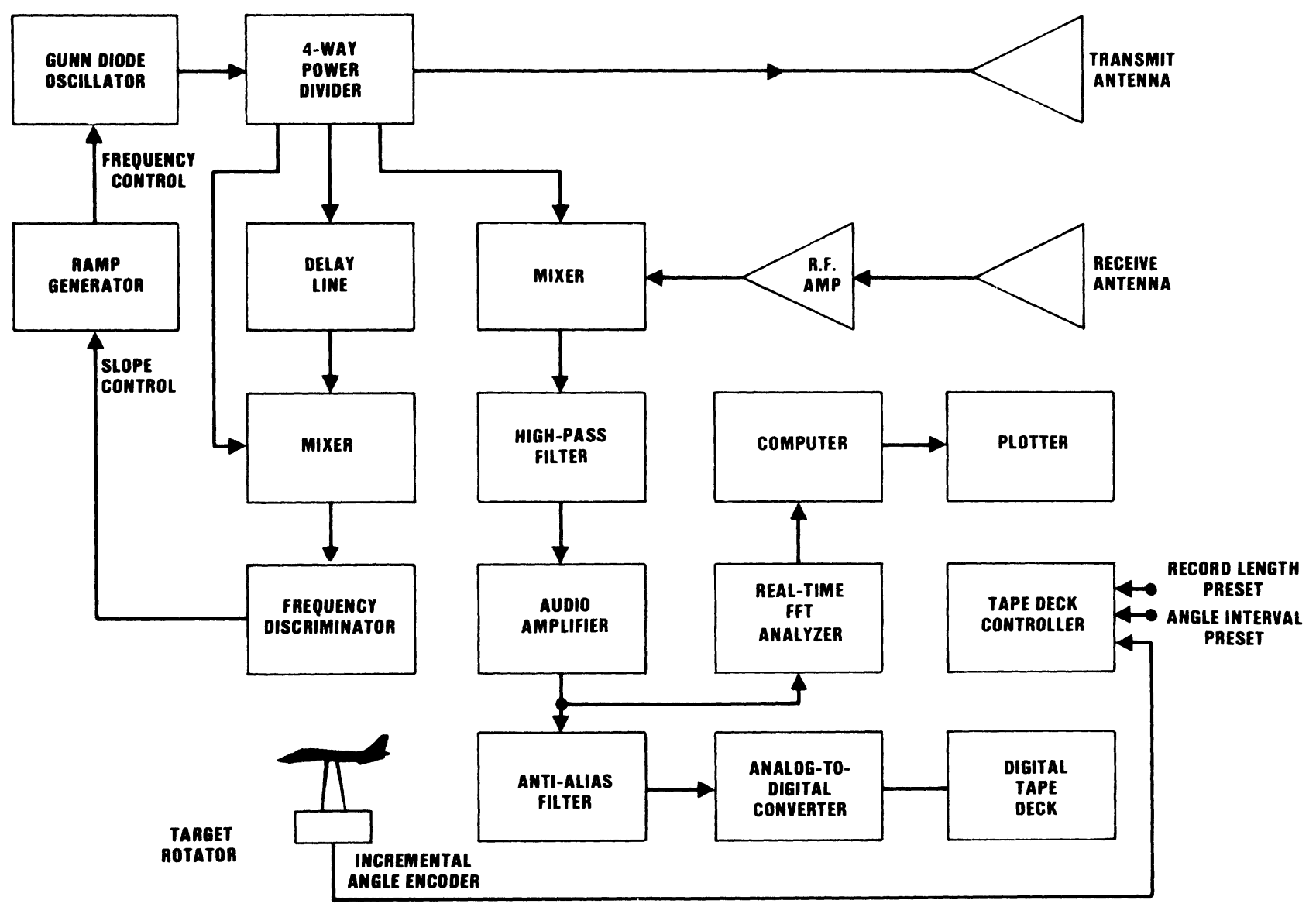

Fig. 2. Block diagram of a linear-FM instrumentation system.

have been developed to produce two-dimensional (range and cross-range) images. The applications involve identification of scatterers for: target signature definition and modeling, determination of RCS of radar antenna systems, RCS reduction, and evaluation of the realism of simulated threat signatures.

Fig. 2 is a block diagram showing the major components of the instrumentation system. The system includes two homodyne receivers in which a sample of the transmitted signal is used directly as a local oscillator (LO) input to a balanced mixer which forms the product of the $\mathrm{LO}$ and received signals. One of the homodyne receivers processes a sample of the transmitted signal passed through a delay line to produce a reference signal. The frequency of the reference mixer output is monitored by a discriminator which provides fine control of the ramp waveform used to modulate the frequency of the RF source. This constitutes a feedback system which linearizes the frequency sweep of the source. A similar homodyne receiver is used to process the received signal after amplification 

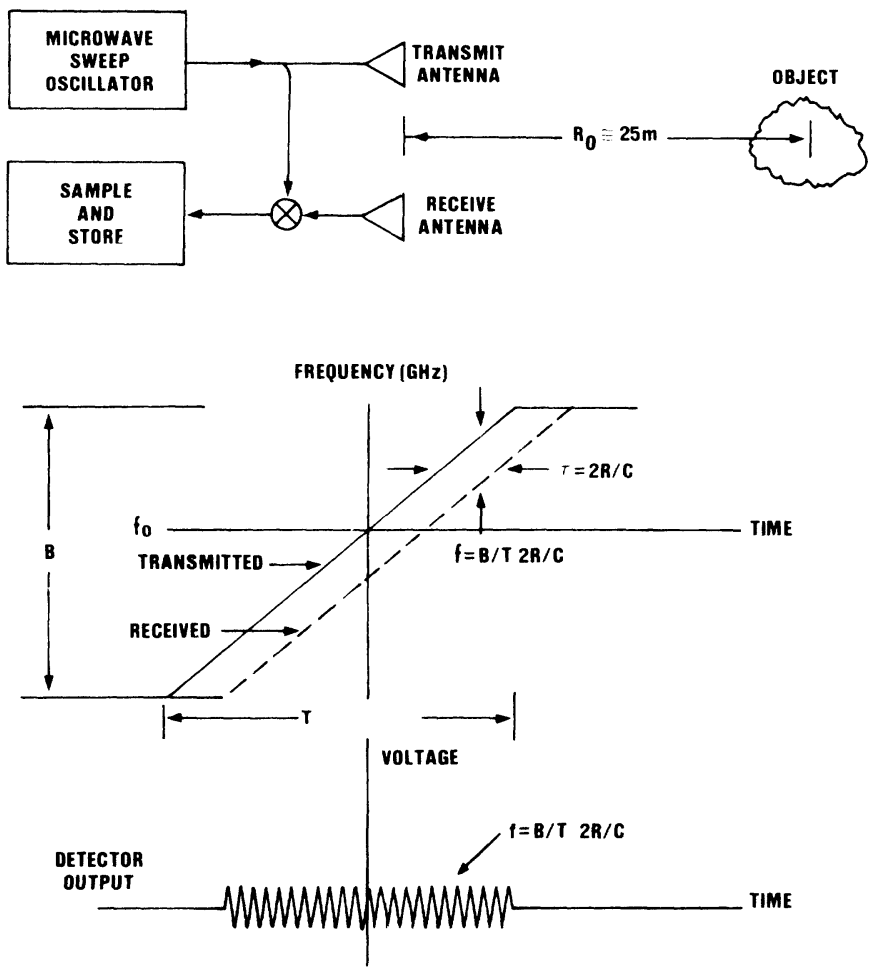

Fig. 3. Simplified block diagram of linear-FM system.

by a low-noise RF amplifier. The antennas are conventional microwave horns and the transmitted power is approximately $10 \mathrm{~mW}$.

The output of the target mixer is conditioned, amplified, and fed to an analog-to-digital converter prior to recording on magnetic tape. The output signal is also fed to a real-time FFT analyzer which samples and digitizes the signal and performs a 1024-point FFT essentially in real-time. The output of the real-time FFT analyzer is passed to a desktop computer which produces annotated, calibrated plots. The real-time FFT analyzer is a Spectral Dynamics, Model 360 and the desktop computer is a Hewlett Packard, Model 9845. The following sections describe the various measurement options afforded by the instrumentation system.

\section{RCS versus Range}

Fig. 3 shows a simplified block diagram of the measurement system which operates by irradiating the entire target with a broad beam from a small-aperture antenna. The instantaneous frequency of the RF carrier is linearly deviated about $f_{0}$ by $+B / 2$ during a period $T$. The transmitted and received signals are mixed in the homodyne receiver to form the product which can be expressed as

$$
e(t)=E \prod(t / T) \cos \left[(4 \pi B R / c T) t+\left(4 \pi f_{0} / c\right) R\right] .
$$

Because the signal frequency is proportional to range, Fourier analysis of the signal provides a measure of range. If the target consists of several reflecting points, the composite will be a linear combination of signals and, by the superposition property, the Fourier transform will accomplish range sorting. By this process, the target space is subdivided into range resolution cells.

The magnitude of the Fourier-transformed signal provides a range profile of the target with a resolution which is determined by the signal duration $T$. The frequency of the signal output from the homodyne receiver associated with a target at range $R$ is

$$
f=2 B R /(c T) \text {. }
$$

The spectral resolution $\Delta f$ of a sinusoid observed over a period $T$ is of the order of

$$
\Delta f=1 / T .
$$

Combining (2) and (3) and associating the spectral resolution $\Delta f$ with the range resolution $\Delta R$, yields the fundamental relation for the range resolution $\Delta R$

$$
\Delta R=c /(2 B) .
$$

The signal output from the homodyne receiver, expressed by (1) is sampled, digitized, and processed by the real-time FFT analyzer which performs a 1024-point FFT. The output of the analyzer consists of discrete samples of the signal spectrum which, after calibration, are plotted to display the RCS of the target as a function of range.

The range response of the system to an ideal point target is a sinc (.) function with resolution given by (4) and range sidelobes $13 \mathrm{~dB}$ below the peak. The range sidelobes are reduced by using the tapered window option of the analyzer. This reduces the range sidelobes to approximately $40 \mathrm{~dB}$ and decreases the resolution to approximately $10 \mathrm{~cm}$.

The rapid processing time of the analyzer produces real-time CRT displays of the range profiles. The RF bandwidth is swept during a period of approximately $100 \mathrm{~ms}$ and the FFT processing time is less than $10 \mathrm{~ms}$. Hard copies of annotated, calibrated, and scaled range responses can be obtained in approximately $15 \mathrm{~s}$. The range responses display RCS as a function of range with resolution of $10 \mathrm{~cm}$ and granularity of $5 \mathrm{~cm}$. The capability of producing plots of RCS versus range in a short time provides a powerful diagnostic tool for locating, identifying, and characterizing individual scatterers on complex targets.

Figs. 4-7 show examples of RCS versus range data obtained from experimental results. The measurements were performed in a tapered anechoic chamber approximately $8-\mathrm{m}$ wide, $5-\mathrm{m}$ high, and 22-m long. Although the performance of such chambers is generally frequency dependent, the variations as a function of frequency over the swept bandwidths used for these measurements are considered negligible. This is due to the relatively high operating frequencies and narrow illumination beamwidths.

Fig. 4 shows a plot of RCS as a function of range for two small, vertical cylindrical rods separated by $25 \mathrm{~cm}$ obtained by using the on-line diagnostic system. The up-range cylinder was 6-cm long with a diameter of $1.9 \mathrm{~cm}$, the down-range cylinder was $10-\mathrm{cm}$ long with a diameter of $1.25 \mathrm{~cm}$. The polarization was horizontal and the swept bandwidth was $3.1 \mathrm{GHz}$ centered on $7.5 \mathrm{GHz}$. The range response was obtained using a tapered window to reduce the level of range sidelobes; this reduced the resolution to $10 \mathrm{~cm}$. The low-level responses are caused by spurious reflections from the anechoic enclosure.

Fig. 5 shows the RCS of a 1 -in-diameter metal sphere. The 


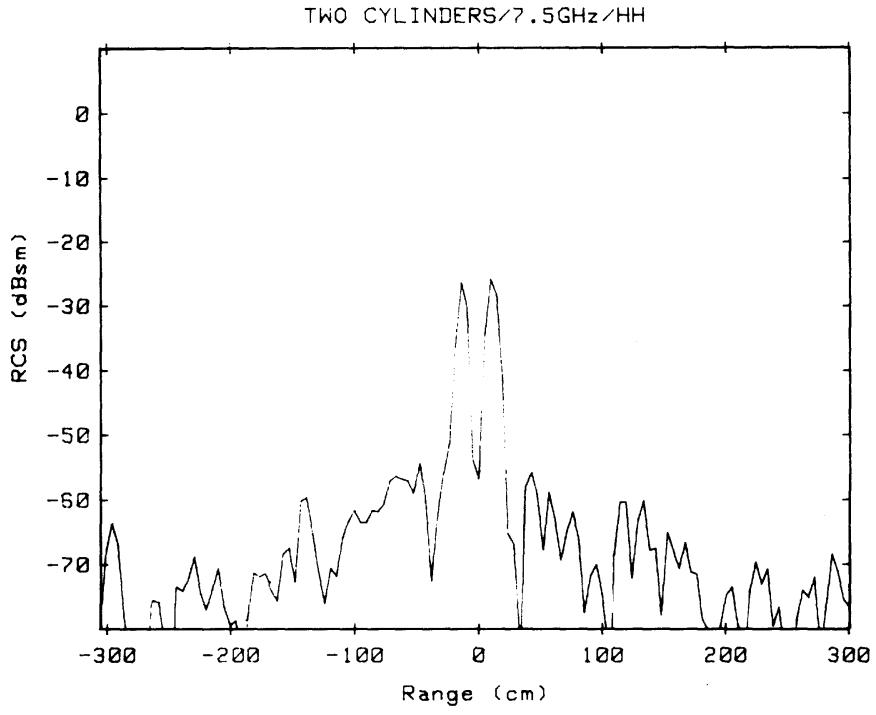

Fig. 4. Range response of two closely spaced cylindrical rods.

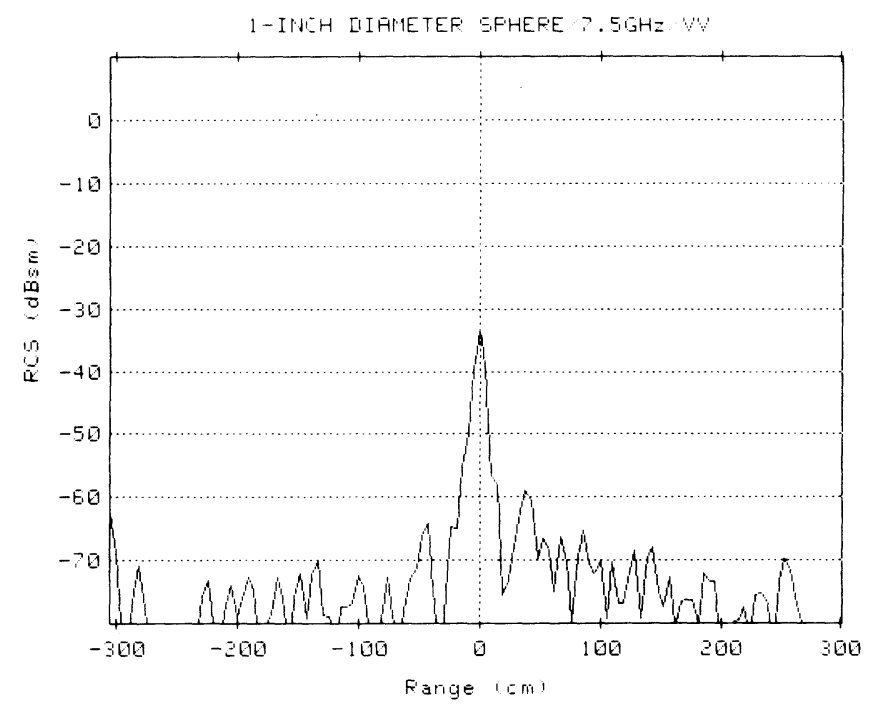

Fig. 5. Range response of a 1-in-diameter sphere.

background level has been reduced by subtracting from the target range profile a similar range profile obtained in the absence of the target. The $-35 \mathrm{dBsm}$ RCS level of the target is clearly measurable in the residual background level of approximately $-70 \mathrm{dBsm}$.

Fig. 6 shows a range response of a square-terminated duct approximately $4 \times 4 \times 18$ in. The duct is essentially a square tube closed at one end and open at the other. The plot is the range response of the duct viewed from the open end using a center frequency of $7.5 \mathrm{GHz}$ and horizontal polarization. The vertical dotted lines represent a range spacing of 18 in corresponding to the length of the duct. Scattering from the duct inlet and from the base are clearly visible.

Fig. 7 shows results of a test similar to that described in the preceding paragraph for a viewing angle of $30^{\circ}$ off the longitudinal axis of the duct. The low-level responses from ranges outside the physical extent of the duct in Figs. 6 and 7 are caused by residues in the cancellation of background signals and by range sidelobe artifacts inherent to the processing.

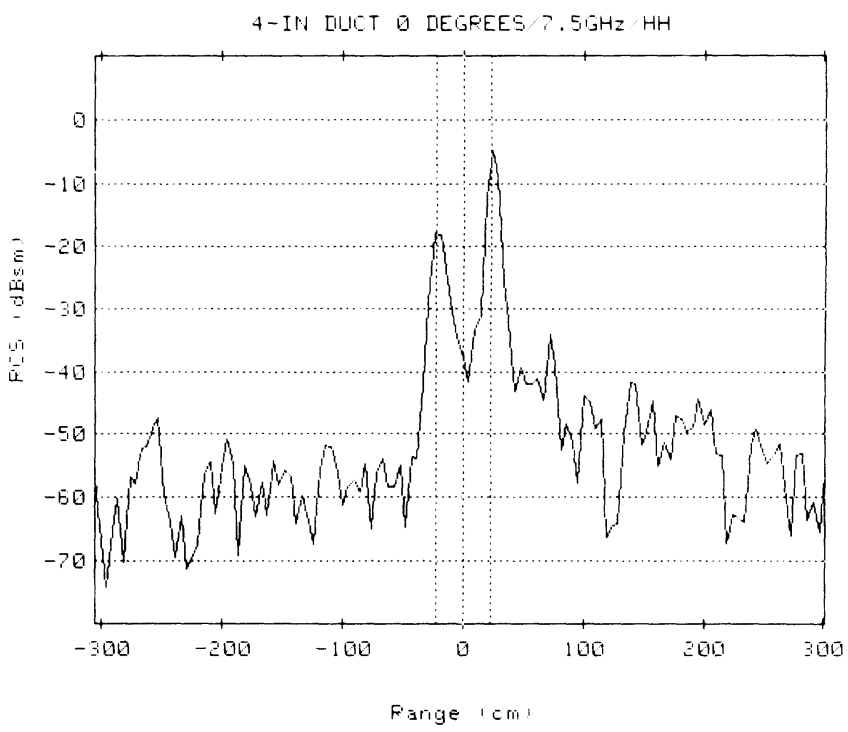

Fig. 6. Range response of a 4 -in square duct; $0^{\circ}$ aspect angle.

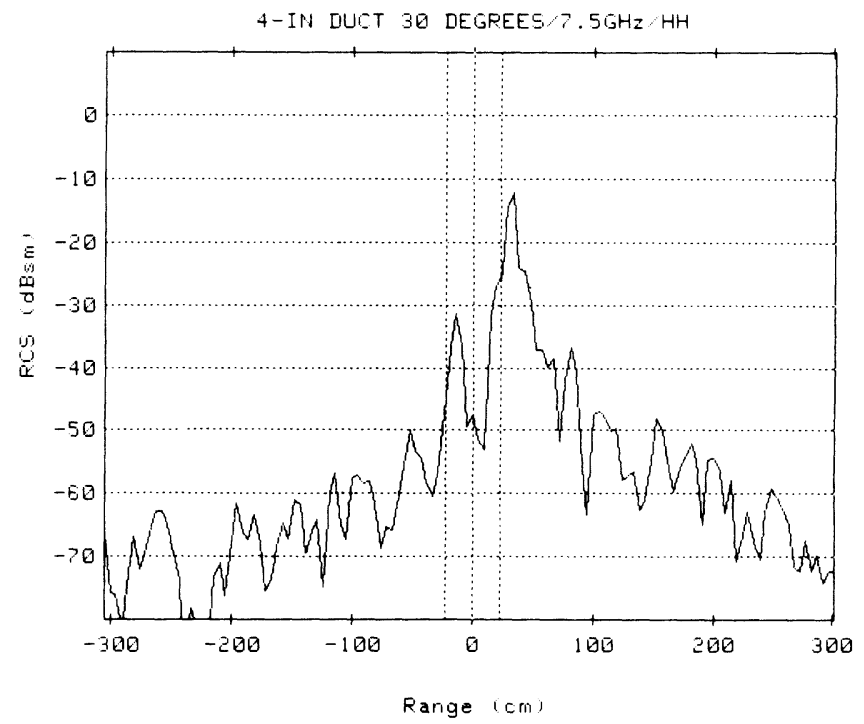

Fig. 7. Range response of a 4-in square duct; $30^{\circ}$ aspect angle.

The more pronounced down-range structure is assumed to be caused by multiple reflections between the ends of the ducts.

\section{RCS versus Frequency}

The output of the homodyne receiver provides a measure of the signal received as a function of the irradiating frequency. In an ideal measurement environment, the variations in the received signal can be attributed to the target and its response as a function of frequency thus determined directly. In practical situations, however, range profiles exhibit spurious responses at ranges other than that of the target. These can result from unintentional echoes from the ground or enclosures, from structures used to support the target, or from signal leakage between the transmitting and receiving antennas. The effects of the spurious echoes on the processed signal must be minimized in order to preserve the fidelity of the target frequency response. The signal processing required to extract the RCS as a function of frequency is diagrammed in Fig. 8, and consists of the following four distinct operations on the 


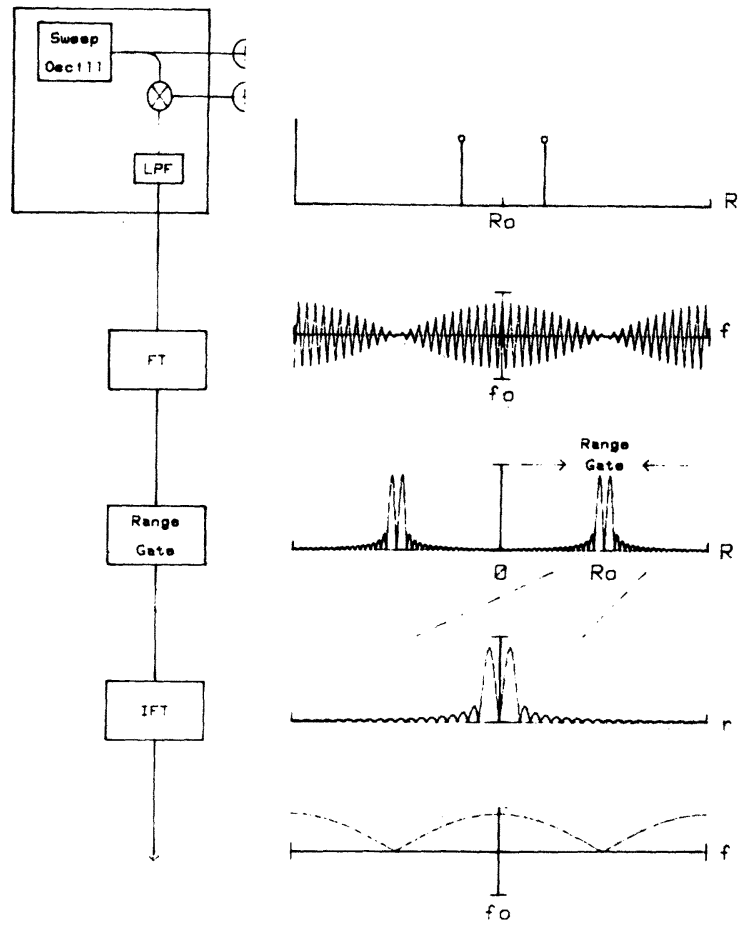

Fig. 8. Signal processing operations for RCS frequency responses.

recorded output signal of the linear-FM system: 1) Fourier transforming the available data to obtain a range profile; 2) applying a range gate centered on $R_{0}$ which retains all elements of the range profile within a known target bound and sets the others to zero; 3 ) translating the range-gated portion of the range profile by $R_{0}$ so that $R_{0}$ becomes zero range; and 4) inverse Fourier transforming the range-gated range profile to recover the complex envelope associated with the target bound. The FFT operation is performed on the output of the homodyne receiver signal by the real-time analyzer. The phase and magnitude of the FFT output are transferred to the memory of the computer which is used to perform the range-gating function, the inverse FFT, the calibration and plotting. Plots of RCS versus frequency can be obtained in approximately $40 \mathrm{~s}$.

Measurements performed on test targets are calibrated by determining their response relative to that of a calibrator with known RCS measured under identical conditions. A calibrator consisting of a metal sphere provides a frequency-independent RCS if the diameter is sufficiently large; other calibration shapes such as cylinders of flat plates can also be used if a correction factor is applied to account for their frequencydependent RCS. The measured frequency responses of targets are subject to variations as a function of frequency of all system components such as antennas, mixers, and transmission lines. Because the frequency response of the instrumentation components is invariant for both test and calibration targets, these effects are removed by the relative measurement in which the target RCS is determined by the ratio of measured responses for target and calibrator. The target frequency responses are calibrated only in terms of magnitude; the phase of the target response as a function of frequency is, therefore, not determined. The determination of the phase

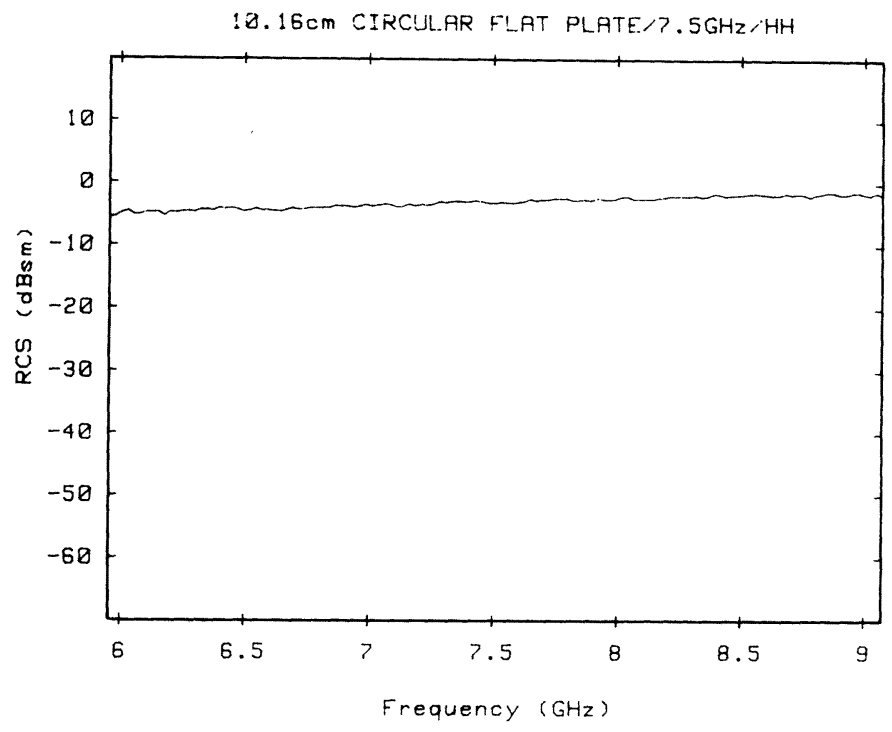

Fig. 9. Frequency response of circular flat plate at normal incidence.

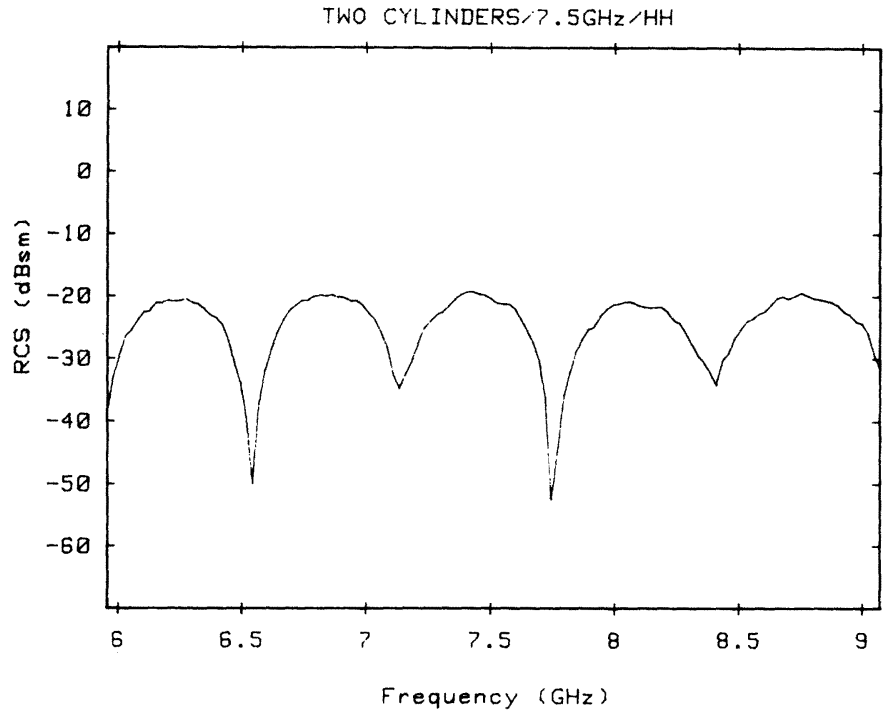

Fig. 10. Frequency response of two closely spaced cylindrical rods.

response would require knowledge of the theoretical phase response of the calibrator. Figs. 9-12 show examples of RCS versus frequency data obtained from experimental results.

Fig. 9 shows the RCS as a function of frequency for a circular plate approximately $10 \mathrm{~cm}$ in diameter viewed at normal incidence. The plot shows the predictable increase in RCS proportional to the square of frequency.

Fig. 10 shows a plot of RCS as a function of frequency for the two cylindrical rods with RCS versus range shown in Fig. 3. The lobes of the frequency response are well correlated with the predicted width of $600 \mathrm{MHz}$. The frequency response consists of 128 values spanning the $3.1-\mathrm{GHz}$ swept bandwidth.

Fig. 11 shows the RCS as a function of frequency for a 1 -indiameter metal sphere. The low-level, rapid variations as a function of frequency are caused by interference between the target response and contribution from the residual echoes of the anechoic chamber. The response shows the proper reso- 

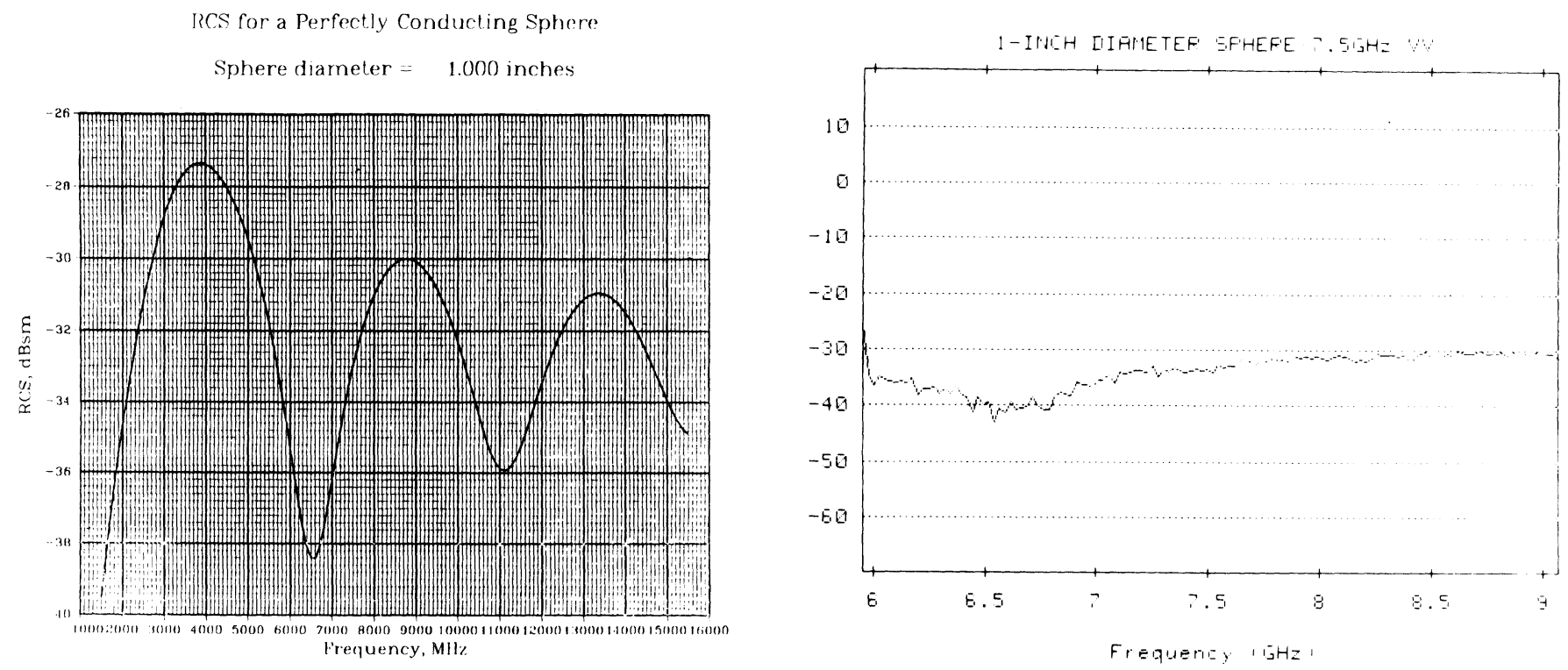

Fig. 11. Frequency response of 1 -in-diameter sphere.

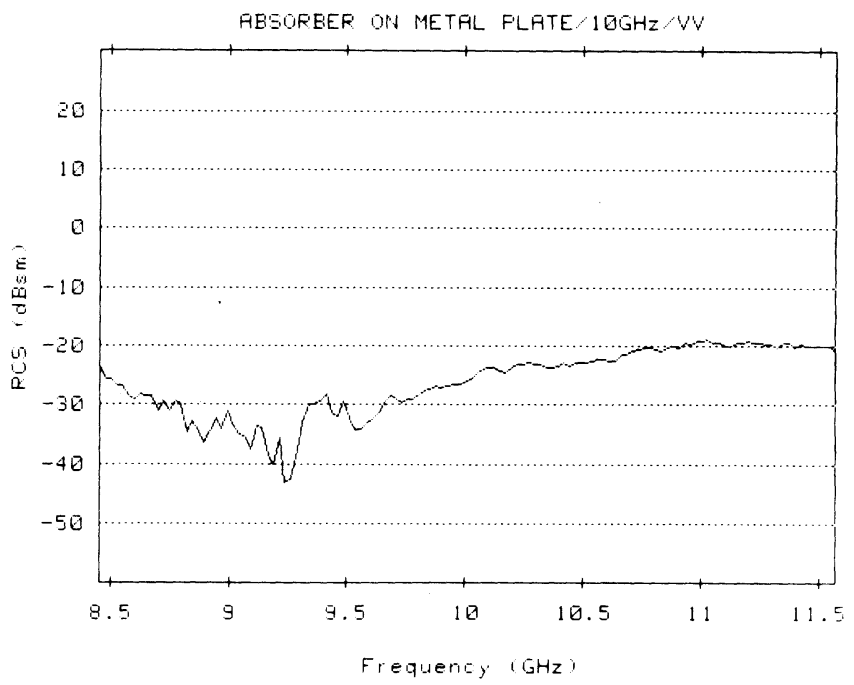

Fig. 12. Frequency response of 1-cm-thick foam absorber.

nant behavior and is well correlated with the theoretical response which dips to $-38.2 \mathrm{dBsm}$ at a frequency of $6.5 \mathrm{GHz}$.

Fig. 12 shows the RCS as a function of frequency for a metal plate covered with a sheet of $1-\mathrm{cm}$-thick radar absorbing material. The response covers a frequency range of $3.1 \mathrm{GHz}$ centered on $10 \mathrm{GHz}$. The response of the metallic plate has been normalized to $0 \mathrm{dBsm}$ and the plot shows the attenuation of the material to be maximum near $9 \mathrm{GHz}$.

\section{RCS Versus Range and Cross Range}

As shown in a preceding section, resolution in range is obtained by spectral analysis of the homodyne receiver output. This analysis separates the target response into range cells. Resolution in cross range is obtained by processing the signal content of each range cell as the target is rotated. For each successive small increment of target rotation, a linear-FM pulse is transmitted and received. The rotation rate is sufficiently slow, relative to the pulse duration, that the target is consid- ered stationary while the pulse is transmitted. After range resolution is obtained for each increment, the signal content of each resolution cell is processed as a function of angular position to extract a Doppler signal which determines the cross-range coordinates. The basis of Doppler processing consists of the observation that the frequency shift of signals reflected by a rotating target point is proportional to the lateral offset of the reflector measured along an axis normal to the axis of rotation and to the line-of-sight. Doppler processing of signals reflected from a two-dimensional target rotating in a plane, therefore, yields resolution in cross range. When range and cross-range processing are combined, twodimensional images can be produced which display the spatial distribution of RCS as a function of range and cross range [3]. The two-dimensional images are produced by an off-line computer which operates on samples of the output of the homodyne receiver which have been digitized and stored on magnetic tape. These data consist of records of the target response for successive angular increments, typically $0.1^{\circ}$, taken over a complete rotation of the target. Two-dimensional images are produced by processing 128 such records. The two-dimensional images are useful for locating scatterers on complex targets which cannot be unambiguously separated on a onedimensional range response.

Figs. 13-17 present two-dimensional images of complex targets consisting of the target RCS as a function of down-range and cross-range coordinates. The size of the target field mapped is $20 \times 20 \mathrm{ft}$ and the resolution is $20 \mathrm{~cm}$. The images are obtained by coherently processing arrays of range profiles over angular rotations of the order of $10^{\circ}$.

Fig. 13 is an isometric display of a two-dimensional image of a BQM-34S target drone viewed in a head-on aspect. An outline of the vehicle, approximately $20 \mathrm{ft}$ in length, is drawn to scale in the proper orientation. The results were obtained from $10-\mathrm{GHz}$ wideband data using vertical polarization. Scattering from the wingtip pods and tail structures resolved in cross range. would not be resolvable in range profiles. Multiple 


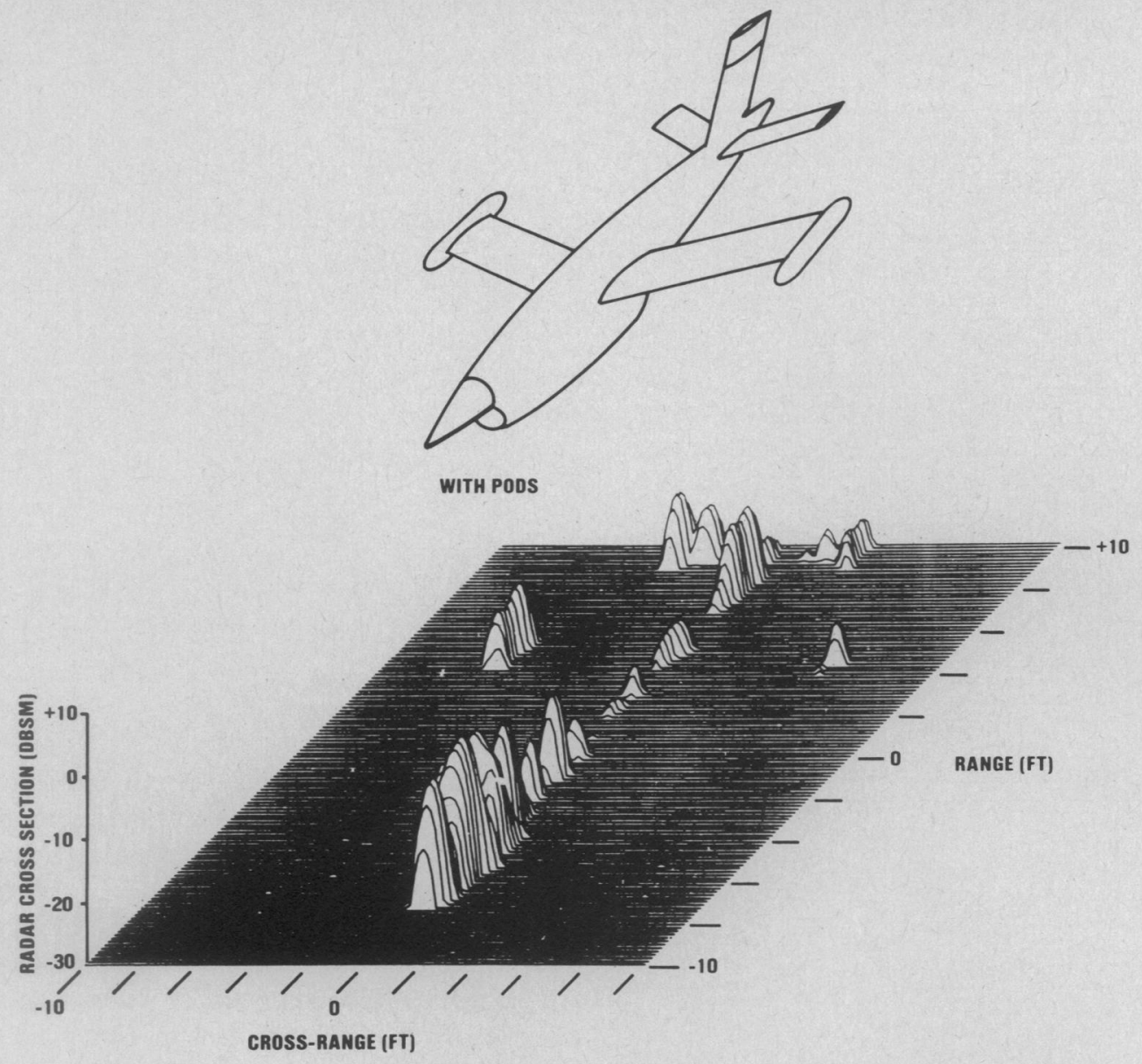

Fig. 13. Two-dimensional image of a drone target vehicle.

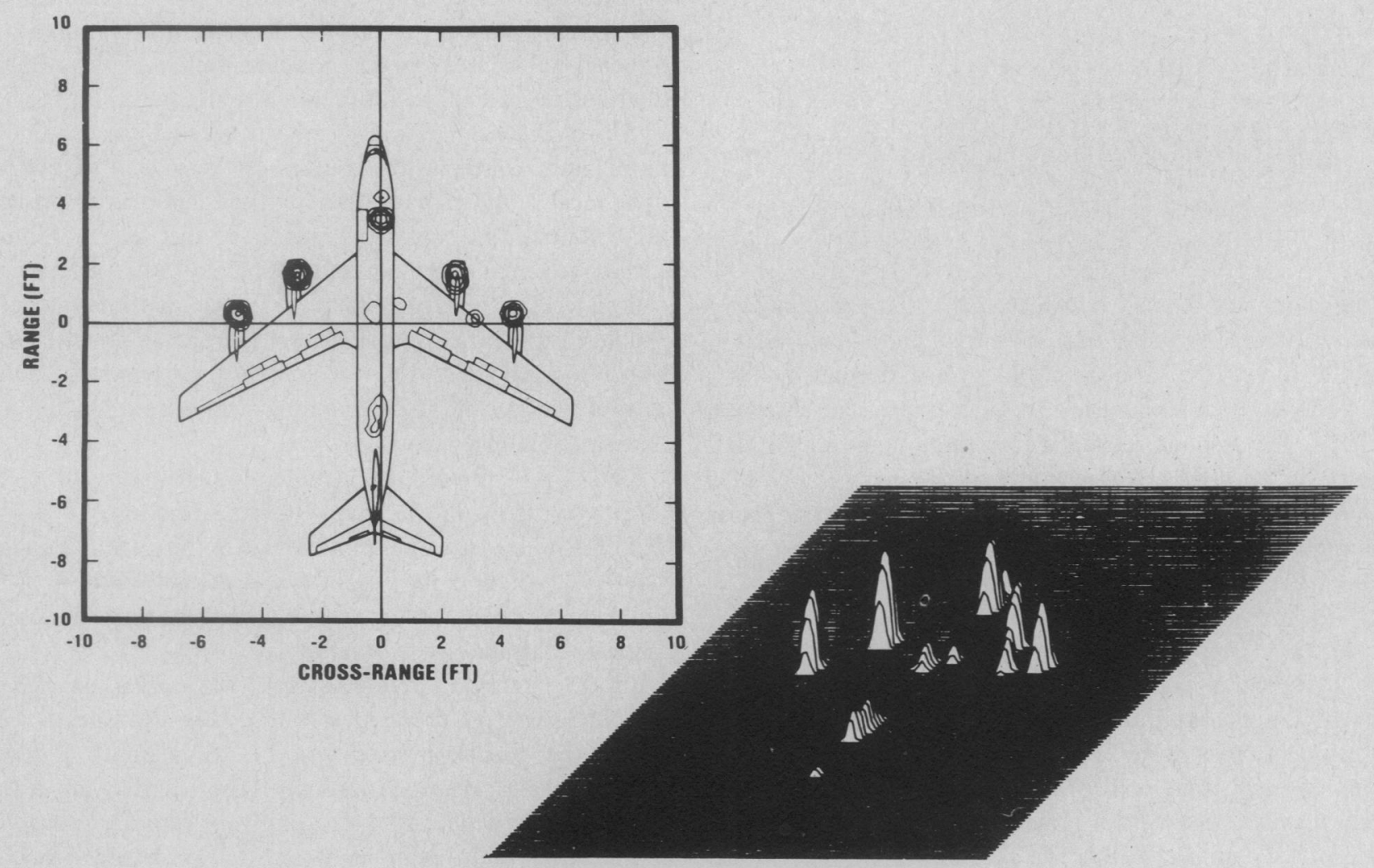

Fig. 14. Two-dimensional images of a scale aircraft model. 

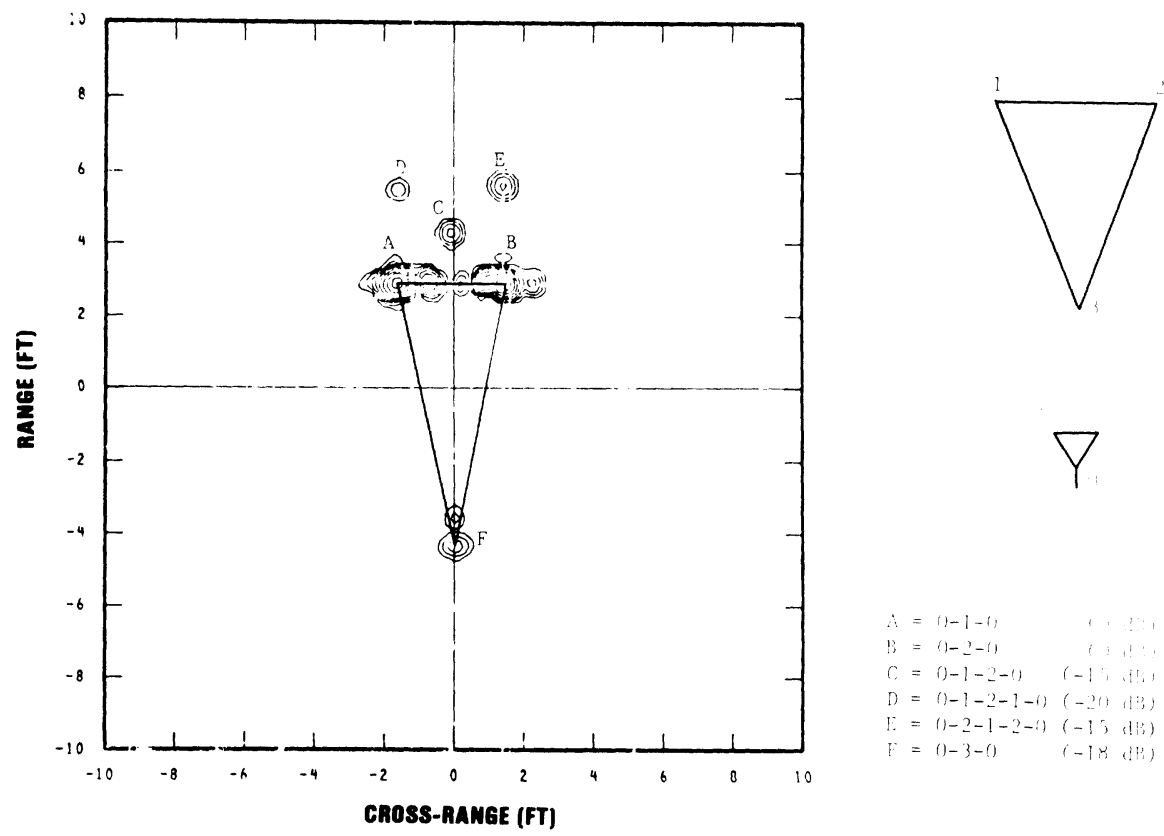

Fig. 15. Two-dimensional image of a vertical wedge.

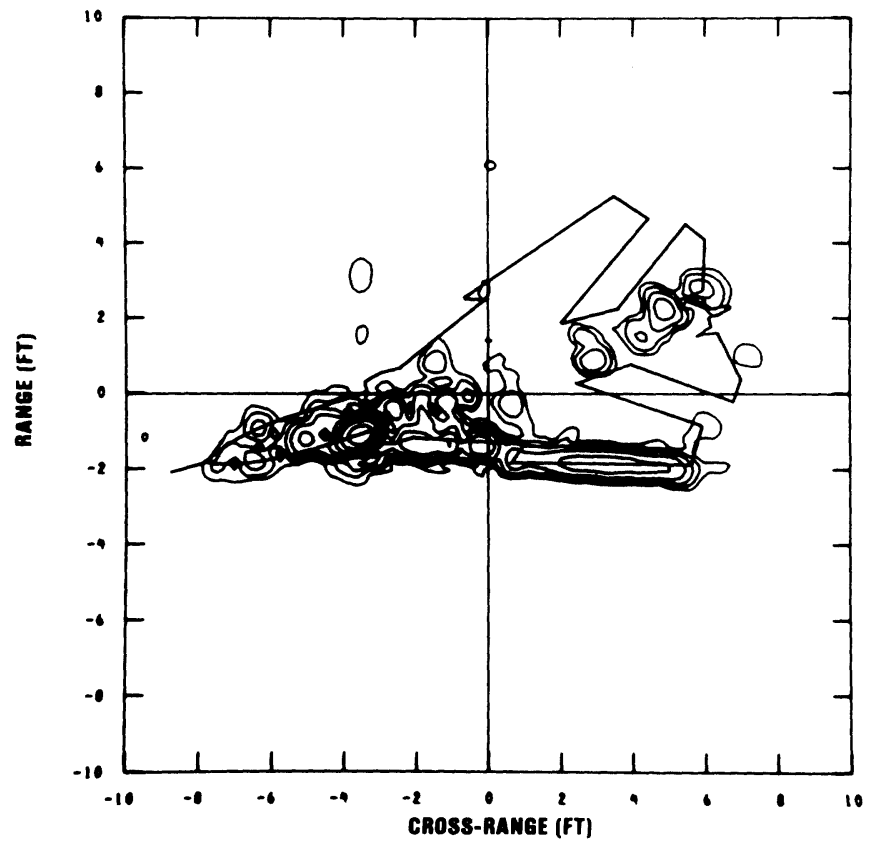

Fig. 16. Two-dimensional image of a scale aircraft model viewed from approximately beam aspect.

responses from the front part of the vehicle are attributed to reverberations from structures internal to a semi-transparent fiberglass cowling making up the front section.

Fig. 14 shows two-dimensional images of a $1: 10$ scale model aircraft, viewed from a tail aspect, obtained from $15-\mathrm{GHz}$ wideband data using vertical polarization. The identical image data are plotted in two formats. The right figure is an isometric display similar to the one shown in the preceding example. The left figure is a contour plot showing lines of constant RCS in $3-\mathrm{dB}$ increments. While the isometric plot effectively displays the relative magnitude of individual scatterers, the contour plot provides graphic association between the location of scatterers and the physical outline of the vehicle.
Fig. 15 is a two-dimensional image of a wedge formed by two vertical planes. The wedge measured approximately $2.2 \mathrm{~m}$ in length and $1 \mathrm{~m}$ in height and width. The results were obtained from $7.5-\mathrm{GHz}$ wideband data using horizontal polarization. Reflections from point $F$ and diffractions from points $A$ and $B$ are properly correlated with the leading and trailing edges. Scattering centers labeled $D$ and $E$ are due to double diffraction from one trailing edge to the other. Because the received signal is time delayed by the round-trip distance between trailing edges, the responses $D$ and $E$ are displaced down range by a distance equal to the separation between trailing edges. The response at location $C$ is due to double diffraction from one trailing edge to the other. Because the effective signal path 


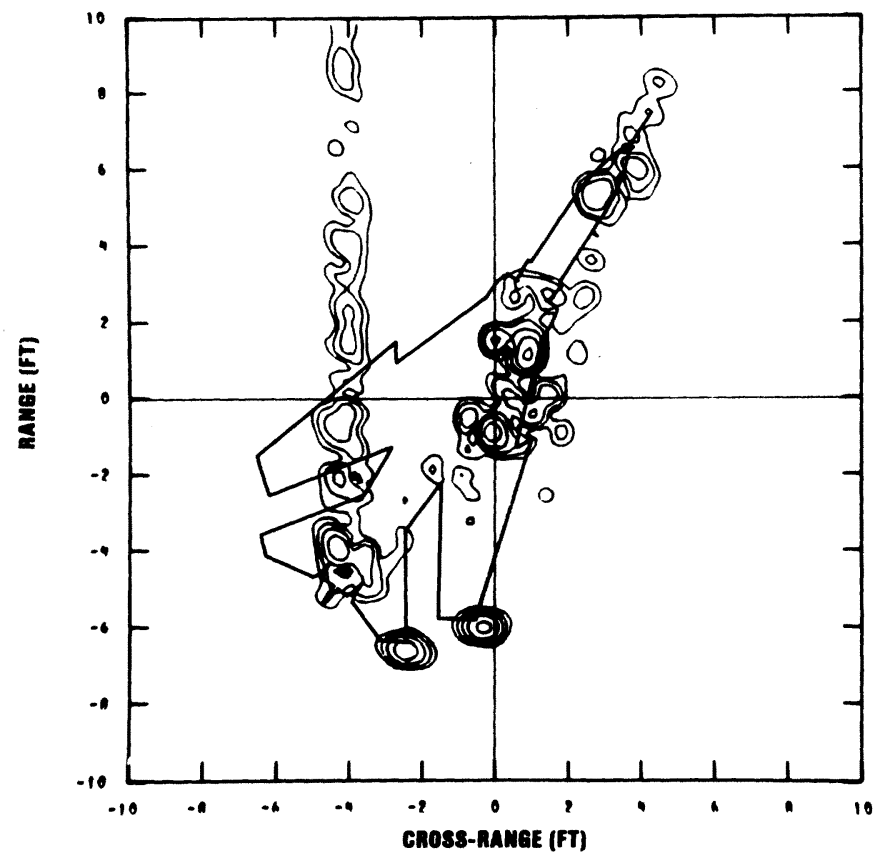

Fig. 17. Two-dimensional image of a scale aircraft model viewed from rear quarter.

length remains constant as a function of rotation, the response properly appears at zero cross range with a range delay equal to one-half of the separation between trailing edges.

Fig. 16 is a two-dimensional image of a $1: 3.5$ scale aircraft model approximately $5 \mathrm{~m}$ in length. The results were obtained from $1.5-\mathrm{GHz}$ wideband data using vertical polarization. The aspect angle placed the line-of-sight normal to the leading edge of the wing.

Fig. 17 is a two-dimensional, contour image of the same aircraft model viewed from a rear quarter aspect. Discrete scattering centers are evident at the tips of the wings and tail. A range-extended response approximately $4 \mathrm{ft}$ to the left of center is due to reverberations occurring within the exhaust duct.

\section{SUMmARY OF CAPABILITIES}

The systems described provide a unique capability for highresolution RCS measurements. The various options of operation provide a powerful diagnostic tool for locating scattering centers on complex targets. The one-dimensional responses of RCS versus range and frequency are produced essentially in real time and hundreds of such responses can be obtained in a working day. By processing digitally recorded data, twodimensional images of the target can be produced. This requires batch processing, typically with a one-day delay at an approximate cost of $\$ 10$ image. Future plans include the expansion of these capabilities to higher operating frequencies in order to allow collection of such data on scale models of targets at frequencies representative of operational radars.

\section{REFERENCES}

[1] C. C. Chen and H. C. Andrews, "Multi-frequency imaging of radar turntable data," IEEE Trans. Aerosp. Electron. Syst., vol. AES-16, pp. 15-22, Jan. 1980.

[2] J. L. Walker, "Range-Doppler imaging of rotating objects," IEEE Trans. Aerosp. Electron. Syst., vol. AES-16, pp. 23-52, Jan. 1980.

[3] D. Mensa, High Resolution Radar Imaging. Dedham, MA: Artech House, 1982. 